\title{
Information Science
}

Franz Plochberger

This science researches all around the term and word Information. 